\title{
Arthroscopic Control for Safe and Secure Seating of Suspensory Devices for Femoral Fixation in Anterior Cruciate Ligament Reconstruction Using Three Different Techniques
}

\author{
Seo Goo Kang, MD and Yong Seuk Lee, MD \\ Department of Orthopaedic Surgery, Seoul National University Bundang Hospital, Seoul National University College of Medicine, Seongnam, Korea
}

\begin{abstract}
Purpose: The purpose of this study was to evaluate the efficacy of our technique that allows direct visualization of seating of suspensory devices in anterior cruciate ligament $(\mathrm{ACL})$ reconstruction.

Materials and Methods: Three different suspensory devices (TightRope RT, RetroButton, and EndoButton) were used in ACL reconstruction using 3 different techniques (outside-in, anteromedial [AM] portal, and transtibial techniques). Positioning of a guiding material and seating pattern of the suspensory devices were evaluated according to the surgical technique and suspensory device used.

Results: On the transtibial technique, 21 of total 26 cases (81\%) of single bundle reconstructions and 22 of total 22 cases (100\%) of double bundle reconstructions required superolateral capsulotomy where buttons were found in 21 of total 21 cases (100\%) and 17 of 22 cases (77\%), respectively. On the AM portal technique, all patients required capsulotomy and the button was found in only 18 of total 32 cases (56\%) even after capsulotomy. On the outside-in technique, all patients required capsulotomy and the button was found in 86 of total 86 cases (100\%).

Conclusions: Our technique for direct visualization of seating of the suspensory devices was more effective in outside-in and single bundle transtibial ACL reconstruction. However, it was less effective in double bundle transtibial and AM portal ACL reconstructions.
\end{abstract}

Keywords: Knee, Anterior cruciate ligament, Suspensory device, Outside-in technique

\section{Introduction}

There are multiple options for femoral fixation of a soft tissue graft in anterior cruciate ligament (ACL) reconstruction. In particular, suspensory femoral fixation has attracted increasing interest with the popularity of outside-in femoral drilling for better replication of the anatomy of the native $\mathrm{ACL}^{1-4)}$. However, some

Received September 20, 2016; Revised October 19, 2016;

Accepted November 7, 2016

Correspondence to: Yong Seuk Lee, MD

Department of Orthopaedic Surgery, Seoul National University Bundang

Hospital, Seoul National University College of Medicine, 82 Gumi-ro

173beon-gil, Bundang-gu, Seongnam 13620, Korea

Tel: +82-31-787-7199, Fax: +82-31-787-4056

E-mail: smcos1@snu.ac.kr

This is an Open Access article distributed under the terms of the Creative Commons Attribution Non-Commercial License (http://creativecommons.org/licenses/by-nc/4.0/) which permits unrestricted non-commercial use, distribution, and reproduction in any medium, provided the original work is properly cited. difficulties could be encountered during ACL reconstruction using suspensory devices: incomplete seating, flipping failure, jamming inside the femoral canal or iliotibial band, and pulling to the outside of the skin or to the joint ${ }^{5-7)}$.

TightRope RT (Arthrex, North Naples, FL, USA) is an adjustable-loop length suspensory fixation device. It is usually used with FlipCutter (Arthrex) that enables bone sparing procedure. Theoretically, this combination allows proper graft-tunnel fit. However, the longer the TightRope loop is, the higher the risk of being pulled too far off or too little $e^{6,8)}$. Up to now, there is insufficient information on the intraoperative difficulties and complications related to this device. The availability of such information is important because surgical difficulties and iatrogenic complications can be avoided if the mechanism of the device is understood and some technical tips are practiced. Previously, we also encountered some difficulties (incomplete seating, pulling at the outside portion of the iliotibial band, or slipping during distal pulling due to incomplete transverse flipping) during flipping. 


\section{Kang and Lee. Arthroscopic Technique for Safe Seating of Suspensory Device in ACL Reconstruction}

We proposed some technical tips and methods for prevention of intraoperative difficulties and complications in ACL reconstruction using similar devices in our previous reports ${ }^{1,6)}$. The purpose of the current study is to evaluate the efficacy of our direct visualization technique in ACL reconstructions performed using 3 different techniques with different suspensory devices. The hypothesis of this study was that safe and secure seating of suspensory devices would be possible using this technique regardless of the surgical technique and suspensory device used in ACL reconstruction

\section{Materials and Methods}

From 2010 to 2015, 3 different suspensory devices (TightRope RT, RetroButton [Arthrex], and EndoButton loop [Smith \& Nephew, Andover, MA, USA]) were utilized in ACL reconstruction performed using 3 different techniques (outside-in technique, anteromedial $[\mathrm{AM}]$ portal technique, and transtibial technique). In ACL reconstructions performed during this period, a pulling Ethibond (Ethicon, Somerville, NJ, USA) or wire was searched around the femoral exit point with an arthroscope. This technique was tried with suitable adjustments in nearly all cases in single bundle ACL reconstruction if the button could not be found in the suprapatellar pouch. In double bundle transtibial reconstruction, this technique was also applied if any of the pulling Ethibond or wire of two bundles could not be found. However, analysis was performed using only surgical records describing this technique.

Firstly, a guiding Ethibond or wire was searched in the suprapatellar pouch with viewing from the anterolateral (AL) portal. Shaking of the guiding Ethibond or wire was performed at the outside portion of the knee if the guiding material was not seen in the suprapatellar pouch area. Superolateral capsulotomy was performed along the guiding material from outside to inside the joint with a shaver and the femoral exit point was confirmed (Fig. 1). This procedure was performed by a single surgeon in all cases. Positioning of the guiding material (necessity of superolateral capsulotomy) and seating pattern of the suspensory devices were analyzed according to the surgical technique and suspensory device used.

\section{Surgical Technique}

Summaries of the 3 different surgical techniques are provided below. Three suspensory devices (TightRope RT, EndoButton, and RetroButton) were used during ACL reconstruction performed using the 3 different techniques. During reconstruction, the focus was on the safe and firm seating of the suspensory devices with arthroscopic control. In all cases, a hamstring autograft was used and the grafts' free ends were sutured with No. 5 Ethibond (approximately $3 \mathrm{~cm}$ in length) regardless of the technique used.

\section{Outside-in Technique}

An arthroscope was inserted via the AM portal and a femoral guide set (RetroConstruction Drill Guide Set, Arthrex) at a $90^{\circ}$ angle was inserted via the AL portal using a FlipCutter drill guide system. The tip of the guide hook was pointed at the posterosuperior corner of the ACL footprint using the outsidein technique. If the footprint was not well noticed, the lateral intercondylar ridge and lateral bifurcate ridge were used as landmarks. Once the FlipCutter tip was in the joint, the drill sleeve was slid back and the femoral guide was removed. The blue hub was turned counterclockwise until it contacted the cross-pin. Using an arthroscopic probe, the blade of the FlipCutter was folded until it was perpendicular to the shaft and the blue hub was turned clockwise until tight. The drill sleeve was pushed down to the bone and then pushed approximately $7 \mathrm{~mm}$. Retro-reaming was then performed until the FlipCutter bottomed out on the drill sleeve. The reaming length was also double checked using a rubber ring. After removing the FlipCutter by pushing it back into the joint and straightening the blade tip, the looped wire was inserted through the drill sleeve and used for graft passage.

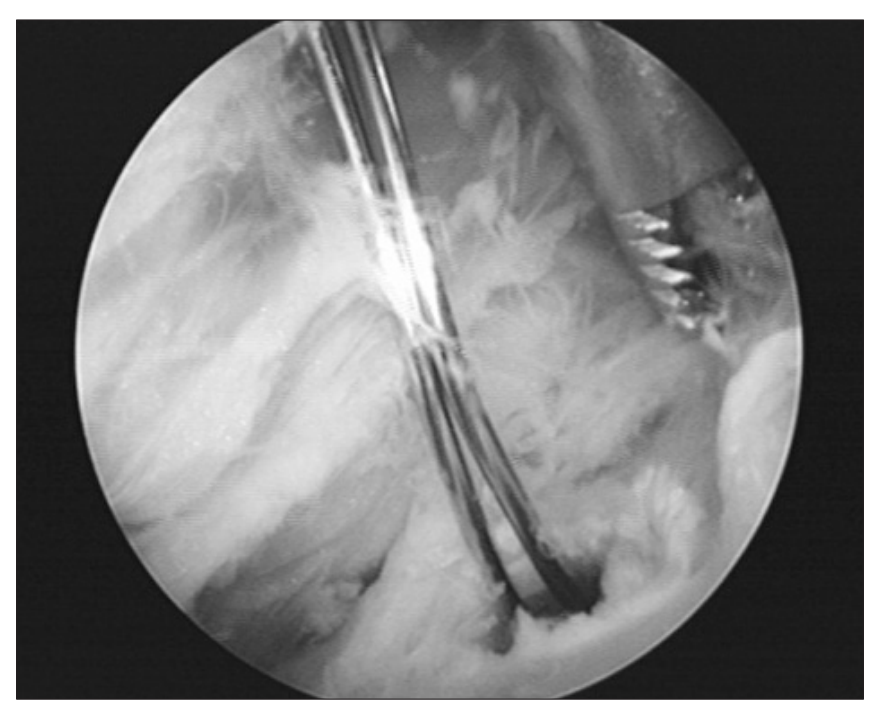

Fig. 1. Superolateral capsulotomy was performed along the guiding material from outside to inside the joint with a shaver. 


\section{Anteromedial Portal Technique}

An arthroscope was inserted via the AL portal in the familiar $90^{\circ}$ knee flexion position. The transportal guide (TPG) was brought through the AM portal and seated in the 10-o'clock position for the right knee and 2-oclock position for the left knee. A beath pin was inserted into the joint through the TPG. The tip of the beath pin was pointed at the central portion of the footprint. If the footprint was not well noticed, the lateral intercondylar ridge and lateral bifurcate ridge were used as landmarks. The beath pin was inserted into the lateral wall of the notch at the centrum of the femoral socket. Then, a cannulated reamer was inserted through the guide wire and reaming was performed. A looped wire was inserted through the beath pin hole and used for graft passage.

\section{Transtibial Technique}

The tibial tunnel was drilled from the proximal and medial parts to lower the obliquity of the femoral tunnel. In general, the starting point of the tibial tunnel is located midway between the posterior cortex of the proximal tibia and the medial margin of the tibial tuberosity. Following the tibial tunnel creation, a transtibial femoral tunnel guide was inserted through it and the rear angle of the guide was placed in the direction of 10-oclock position for the right knee and 2-oclock position for the left knee in a more than $100^{\circ}$ flexion. Then, a tunnel with a depth of $30 \mathrm{~mm}$ was drilled to achieve a posterior wall thickness of approximately 1-2 $\mathrm{mm}$. Then, a looped wire was inserted through the beath pin hole and used for graft passage. To make the posterolateral bundle for double bundle reconstruction, the knee was held in $90^{\circ}$ flexion and the ACL guide was inserted at an angle of $55^{\circ}$ via the AM portal. The guide tip was pointed to the most posterolateral aspect of the footprint. Then, the guide pin was advanced to the femoral footprint, which was located below the intercondylar ridge and anterior to the bifurcate ridge ${ }^{9-11)}$.

\section{Superolateral Capsulotomy}

This technique was applied regardless of the reconstruction method. The size of the capsulotomy was approximately $3 \mathrm{~cm}$ and it was mostly healed at the second-look arthroscopy. Under direct visualization through an arthroscope introduced from the AL portal to the superolateral pouch, we shook the inserted wire up and down until we could identify the correct location of guide wire. Then, an arthroscopic shaver was inserted via a skin incision for the FlipCutter or beath pin, and debridement of soft tissue around the guide wire was performed. Then, the tunnel exit was exposed for complete seating without impingement of soft tissue. Debridement was performed up to the convex lateral epicondylar area and it did not advance downward to prevent a neurovascular injury.

The graft was passed from the tibial tunnel to the femoral tunnel and the buttons of suspensory devices were observed outside of the femoral tunnel using the arthroscope. Then, careful flipping was performed under direct arthroscopic visualization (Fig. 2). A probe or a knot-pusher could be used for flipping and compressing the button to the bony surface while maintaining complete flipping. After complete flipping, distal pulling was performed for tensioning. The flipping loop was tied over suspensory devices using a non-sliding knot in the case of TightRope RT (Fig. 3).

\section{Statistical Analysis}

The SPSS ver. 18.0 (SPSS Inc., Chicago, IL, USA) was used. A $\mathrm{p}<0.05$ was considered significant. The Kruskal-Wallis test was used to compare the efficacy of the superolateral capsulotomy among three groups. The Mann-Whitney $U$ test was used as a post hoc analysis to determine significant differences among the groups.

\section{Results}

Eighty-seven TightRope RTs, 78 EndoButtons, and 23 RetroButtons were used for suspensory fixation in ACL reconstruction. All TightRope RTs and RetroButtons were used in ACL reconstruction using the outside-in technique $(n=86)$. All EndoBut-

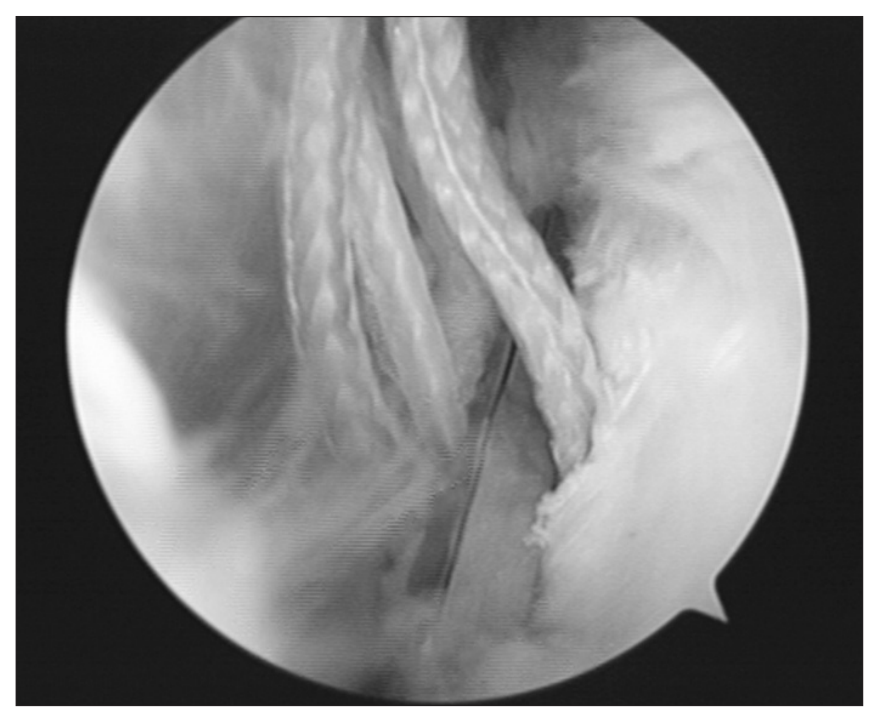

Fig. 2. Flipping of a button was performed under direct arthroscopic visualization. 


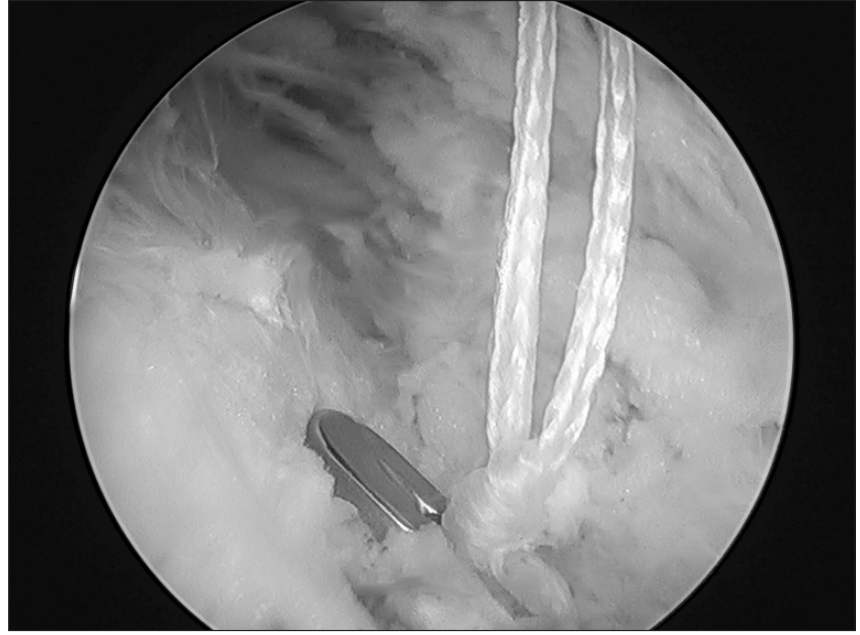

Fig. 3. The Flipping loop was tied over the suspensory device in a nonsliding knot in the case of TightRope RT.

tons were used in the transtibial single bundle or double bundle $\mathrm{ACL}$ reconstruction $(\mathrm{n}=48)$ and $\mathrm{AM}$ portal ACL reconstruction $(\mathrm{n}=32)$.

On the transtibial technique, 21 of total 26 cases $(81 \%)$ of the single bundle reconstructions and 22 of total 22 cases $(100 \%)$ of the double bundle reconstructions required superolateral capsulotomy. Buttons were found in 21 of total 21 cases (100\%) of the single bundle reconstructions and in 17 of total 22 cases (77\%) of the double bundle reconstructions. Five of total 22 cases $(23 \%)$ of the buttons of the posterolateral bundle could not be found even after the superolateral capsulotomy. On the AM portal technique, all patients required capsulotomy and the button was found in only 18 of total 32 cases (56\%) even after capsulotomy. On the outside-in technique, all patients required capsulotomy and the button was found in 86 of total 86 cases (100\%) (Table 1). There was no complication or reoperation related to the techniques.

There was a statistically significant difference in the efficacy of our technique among groups $(\mathrm{p}=0.002)$. In the post hoc analysis, there was a statistically significant difference between single bundle and double bundle transtibial ACL reconstructions $(\mathrm{p}=0.022)$. There was also a statistically significant difference between single bundle transtibial ACL reconstruction and AM portal ACL reconstruction $(\mathrm{p}=0)$. However, there was no statistically significant differences between single bundle transtibial ACL reconstruction and reconstruction using the outside-in technique $(\mathrm{p}=1)$. There was no statistically significant difference between double bundle $\mathrm{ACL}$ reconstruction and AM portal reconstruction $(\mathrm{p}=0.115)$. However, there were statistically significant differences between double bundle ACL reconstruction and reconstruction using the
Table 1. Requirement of Capsulotomy and the Rate of Finding Buttons after Capsulotomy According to Each Technique

\begin{tabular}{lccc}
\hline Techniques & $\begin{array}{c}\text { No. of } \\
\text { cases }\end{array}$ & $\begin{array}{c}\text { Requirement of } \\
\text { capsulotomy }\end{array}$ & $\begin{array}{c}\text { Rate of finding buttons } \\
\text { after capsulotomy }\end{array}$ \\
\hline $\begin{array}{c}\text { Transtibial } \\
\text { Single bundle }\end{array}$ & 26 & $21(81)$ & $21(100)$ \\
$\quad \begin{array}{l}\text { Double bundle } \\
\text { Anteromedial }\end{array}$ & 22 & $22(100)$ & $17(77)$ \\
portal & 32 & $32(100)$ & $18(56)$ \\
Outside-in & 86 & $86(100)$ & $86(100)$ \\
\hline
\end{tabular}

Values are presented as number (\%).

outside-in technique $(\mathrm{p}=0)$ and between reconstructions using the AM portal technique and the outside-in technique $(\mathrm{p}=0)$.

\section{Discussion}

The purpose of this study was to evaluate the efficacy of our flipping under direct visualization technique in ACL reconstructions performed using 3 different techniques and 3 different suspensory devices. The principal findings of this study were that this technique was more effective for the outside-in technique and single bundle transtibial technique. However, it was less effective for the AM portal technique and double bundle transtibial technique, especially in searching the button of the posterolateral bundle. Therefore, the clinical relevance of this study is that this technique is recommended for safe and secure seating of a suspensory device, especially in single bundle transtibial ACL reconstruction and ACL reconstruction using the outside-in technique. In contrast, in some cases of the PL bundle of double bundle ACL reconstruction and AM portal reconstruction, this technique could not be used because it was difficult to find the exit point. Thus, the difference in the efficacy of this technique may be related to the exit point of each ACL reconstruction technique. In the case of the single bundle transtibial and outside-in technique, the exit point is positioned in the relatively anterior area. However, in our opinion, in the PL bundle in the transtibial double bundle reconstruction and AM portal reconstruction, the exit point cannot be positioned in the relatively posterior area due to the proximity to the peroneal nerve and difficulty to access through the AL portal (Fig. 4).

There are 3 different fixation mechanisms for femoral fixation: compression, expansion, and suspension. The last of the three was further divided into the cortical, cancellous, and corticocancellous suspension mechanisms ${ }^{2,12)}$. Suspensory fixation has been reported to result in a higher incidence of tunnel widening ${ }^{13)}$. 

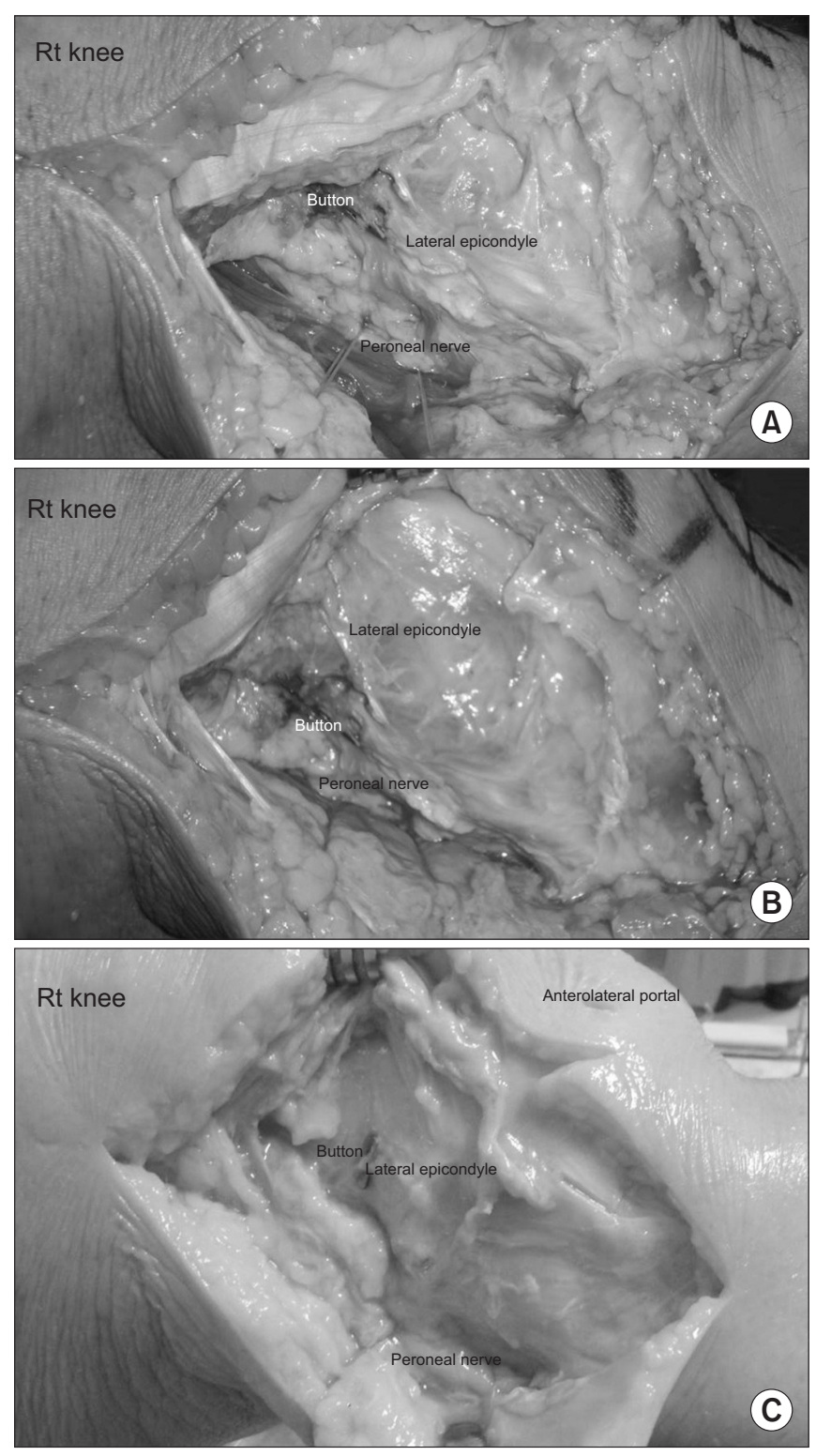

Fig. 4. Button position in each technique and the tagged area is the peroneal nerve. (A) Transtibial technique, (B) anteromedial portal technique, (C) outside-in technique. Rt: right.

On the other hand, it is increasingly used with the popularity of outside-in femoral drilling for better replication of the anatomy of the native ACL and the development of new strong devices ${ }^{3-55}$. However, using these devices, some difficulties could be encountered during the operation such as incomplete seating, flipping failure, jamming inside the femoral canal or iliotibial band, and pulling to the outside of the skin or to the joint. In our previous experience, these kinds of problems could be avoided by observing the fixation directly ${ }^{1)}$. Therefore, it is believed that direct viewing and control is the best way to perform complete and safe fixation.

In our direct visualization technique, only the routine AL portal and an incision for FlipCutter were used and an arthroscope was inserted through the AL portal. Therefore, another skin incision was not required. Seating was controlled by a probe or a knotpusher. The space observed from the AL portal appeared similar to the bursal space in shoulder arthroscopy. In this situation, suture material or wire for graft passage was used as a guide and easy access to the femoral hole along the wire could be obtained. This technique has two important advantages. First, complete seating of a fixation device can be confirmed with arthroscopic visualization. Second, control of the seating is possible using accessory devices such as a probe or a knot pusher. On the other hand, the lateral side of the suprapatellar pouch is open, extra operative time is required, and some extravasation of fluid is inevitable.

There are some limitations of this study. First, the number of patients was different in each group. Second, evaluation was only performed using surgical records. Therefore, due to the limited availability of information, we could not describe more details on the exact size of capsulotomy and the efficacy of the technique for each bundle in double bundle ACL reconstruction. Third, there were some missing data between 2010 and 2012 on whether this technique was used or not. Therefore, it was impossible to identify the exact percentage of enrollment among all ACL reconstructions.

\section{Conclusions}

Direct viewing technique to confirm secure seating of the suspensory devices was more effective for the outside-in and single bundle transtibial technique. However, it was less effective for the double bundle transtibial technique and AM portal technique. For most of the reconstruction techniques, superolateral capsulotomy was required.

\section{Conflict of Interest}

No potential conflict of interest relevant to this article was reported.

\section{References}

1. Lee YS, Ahn JH, Kim JG, Park JH, Park JW, Kim CB, Lee SW. Analysis and prevention of intra-operative complications of TransFix fixation in anterior cruciate ligament reconstruc- 
tion. Knee Surg Sports Traumatol Arthrosc. 2008;16:639-44.

2. Lee YS, Kim SK, Park JH, Park JW, Wang JH, Jung YB, Ahn $\mathrm{JH}$. Double-bundle anterior cruciate ligament reconstruction using two different suspensory femoral fixation: a technical note. Knee Surg Sports Traumatol Arthrosc. 2007;15:1023-7.

3. Alentorn-Geli E, Samitier G, Alvarez P, Steinbacher G, Cugat R. Anteromedial portal versus transtibial drilling techniques in ACL reconstruction: a blinded cross-sectional study at two- to five-year follow-up. Int Orthop. 2010;34:747-54.

4. Chang CB, Yoo JH, Chung BJ, Seong SC, Kim TK. Oblique femoral tunnel placement can increase risks of short femoral tunnel and cross-pin protrusion in anterior cruciate ligament reconstruction. Am J Sports Med. 2010;38:1237-45.

5. Yanmis I, Tunay S, Oguz E, Yildiz C, Ozkan H, Kirdemir V. Dropping of an EndoButton into the knee joint 2 years after anterior cruciate ligament repair using proximal fixation methods. Arthroscopy. 2004;20:641-3.

6. Lee YS, Lee BK, Chun DI. Flipping method of a RetroButton during ACL reconstruction with outside-in femoral drilling using a FlipCutter. Orthopedics. 2012;35:861-4.

7. Muneta T, Yagishita K, Kurihara Y, Sekiya I. Intra-articular detachment of the Endobutton more than 18 months after anterior cruciate ligament reconstruction. Arthroscopy. 1999;15:775-8.

8. Sonnery-Cottet B, Rezende FC, Martins Neto A, Fayard JM,
Thaunat M, Kader DF. Arthroscopically confirmed femoral button deployment. Arthrosc Tech. 2014;3:e309-12.

9. Forsythe B, Kopf S, Wong AK, Martins CA, Anderst W, Tashman S, Fu FH. The location of femoral and tibial tunnels in anatomic double-bundle anterior cruciate ligament reconstruction analyzed by three-dimensional computed tomography models. J Bone Joint Surg Am. 2010;92:1418-26.

10. Kopf S, Forsythe B, Wong AK, Tashman S, Anderst W, Irrgang JJ, Fu FH. Nonanatomic tunnel position in traditional transtibial single-bundle anterior cruciate ligament reconstruction evaluated by three-dimensional computed tomography. J Bone Joint Surg Am. 2010;92:1427-31.

11. Zantop T, Wellmann M, Fu FH, Petersen W. Tunnel positioning of anteromedial and posterolateral bundles in anatomic anterior cruciate ligament reconstruction: anatomic and radiographic findings. Am J Sports Med. 2008;36:65-72.

12. Milano G, Mulas PD, Ziranu F, Piras S, Manunta A, Fabbriciani C. Comparison between different femoral fixation devices for ACL reconstruction with doubled hamstring tendon graft: a biomechanical analysis. Arthroscopy. 2006;22:660-8.

13. Kamelger FS, Onder U, Schmoelz W, Tecklenburg K, Arora R, Fink C. Suspensory fixation of grafts in anterior cruciate ligament reconstruction: a biomechanical comparison of 3 implants. Arthroscopy. 2009;25:767-76. 